\title{
Vagus Nerve Disorder, CTCAE
}

National Cancer Institute

\section{Source}

National Cancer Institute. Vagus Nerve Disorder, CT CAE. NCI Thesaurus. Code C143927.

A disorder characterized by dysfunction of the vagus nerve (tenth cranial nerve). 